\title{
A method for solving fully fuzzy transportation problem with generalized quadrilateral fuzzy numbers
}

\author{
D. Stephen Dinagar ${ }^{1 *}$ and B. Christopar Raj ${ }^{2}$
}

\begin{abstract}
In this paper, we proposed a method to solve fully fuzzy transportation problem by assuming that a decision maker is uncertain about the precise values of the supply, demand and transportation costs. In the proposed method the supply, demand and transportation costs are represented by generalized quadrilateral fuzzy numbers. A numerical example is given to illustrate the proposed method. Decision makers may easily understand and apply in real life transportation problems.
\end{abstract}

Keywords

Ranking function, Fully Fuzzy Transportation Problem, Generalized Quadrilateral Fuzzy Number (GQFN).

\section{AMS Subject Classification}

90C08, 90C90.

${ }^{1} P G$ and Research Department of Mathematics, TBML College, Porayar-609307, India.

${ }^{2}$ Department of Mathematics, Bharathiyar College of Engineering and Technology, Karaikal-609609, India.

*Corresponding author: ${ }^{*}$ dsdina@rediffmail.com; ${ }^{2}$ christofer2010@gmail.com

Article History: Received 21 December 2018; Accepted 11 February 2019

(C)2019 MJM.

\section{Contents}

1 Introduction 24

2 Preliminaries 25

2.1 Fuzzy Set . . . . . . . . . . . . . . 25

2.2 Convex fuzzy set . . . . . . . . . . . . . . . 25

2.3 Fuzzy Number . . . . . . . . . . . . . . . . 25

2.4 Trapezoidal fuzzy number . . . . . . . . . . . . . 25

3 Generalized Quadrilateral Fuzzy Number (GQFN) . 25

3.1 Arithmetic Operations on GQFN . . . . . . . . . 25

3.2 Ranking function for GQFN . . . . . . . . . . . . 26

4 Fully Fuzzy Transportation Problem .26

4.1 Algorithm for solving fully fuzzy Transportation prob-

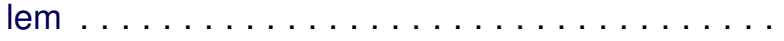
26

5 Numerical Illustration 26

6

Conclusion 27

References

\section{Introduction}

Transportation problem deals with the shipping of a single commodity from various sources of supply to various destinations of demand in which the total transportation cost is to be minimized. In order to solve a transportation problem the decision parameters such as supply, demand and unit transportation cost must be presented as crisp values. But in real life situation the supply, demand and unit transportation cost may not be certain due to several factors. To deal the imprecise data, fuzzy numbers were introduced.

The concept of fuzzy sets were introduced by Zadeh [11] in 1965. Zimmerman [9] formulated the fuzzy linear programming. Transportation problem is a sub-class of linear programming problem. P. Pandian and G. Natarajan [6] proposed a new algorithm to solve fuzzy transportation problem. A. Nagoor Gani and K. Abdul Rajak [4] have presented a fuzzy solution for a two stage cost minimizing fuzzy transportation problem in which supply and demand are trapezoidal fuzzy numbers using a parametric approach. D. Stephen Dinagar and D. Abirami [8] introduced a new arithmetic operations for interval valued fuzzy numbers. Amarpreet Kaur and Amit Kumar [1] proposed a new method for solving fuzzy transportation problems by assuming that a decision maker is uncertain 
about the precise values of the transportation cost, but certain about the supply and demand of the product. M. Venkatachalapathy and A. Edward Samuel [3] presented an Alternative Method (AM) for solving Fuzzy transportation problems by assuming that a decision maker is uncertain about the precise values of the transportation cost only. The proposed method is to solve fully fuzzy transportation problem by assuming supply, demand and unit transportation cost as generalized quadrilateral fuzzy numbers.

In this paper, section 2 gives the basic notions, which are useful to the work. In section 3, the new fuzzy number namely Generalized Quadrilateral Fuzzy Number is proposed with its arithmetic operations. An algorithm is presented in section 4. A relevant illustration is given in section 5. Finally, a conclusion part is also included in section 6 .

\section{Preliminaries}

In this section, some basic definitions and arithmetic operations are reviewed.

\subsection{Fuzzy Set}

A fuzzy set $\tilde{A}=\left\{\left(x, \mu_{A}(x), x \in A, \mu_{A}(x) \in[0,1]\right\}\right.$.

In this pair $\left\{\left(x, \mu_{A}(x)\right\}\right)$, the first element $x$ belongs to the classical set $A$ and the second element $\mu_{A}(x)$ belongs to the interval $[0,1]$, called membership function.

\subsection{Convex fuzzy set}

A fuzzy set $\tilde{A}$ is convex if $\mu_{\tilde{A}}\left(\lambda x_{1}+\left(1-\lambda x_{2}\right)\right) \geq \min \left(\mu_{\tilde{A}}(x)\right.$, $\left.\mu_{\tilde{A}}(x)\right), x_{1}, x_{2} \in X$ and $\lambda \in[0,1]$. Alternatively, a fuzzy set is convex, if all $\alpha$ - level sets are convex.

\subsection{Fuzzy Number}

A fuzzy set $\tilde{A}$ on $\mathrm{R}$ must possess at least the following three properties to qualifys a fuzzy number:

(i) $\tilde{A}$ must be a normal fuzzy set;

(ii) $\tilde{A}$ must be a convex fuzzy set;

(iii) $\tilde{A}$ must be closed and bounded.

\subsection{Trapezoidal fuzzy number}

A fuzzy number $\tilde{A}=(\mathrm{a}, \mathrm{b}, \mathrm{c}, \mathrm{d})$ is said to be trapezoidal fuzzy number if its membership function is given by

$$
\mu_{\tilde{A}}(x)= \begin{cases}\frac{(x-a)}{(b-a)}, & a \leq x \leq b \\ 1, & b \leq x \leq c \\ \frac{(x-d)}{(c-d)}, & c \leq x \leq d\end{cases}
$$

\section{Generalized Quadrilateral Fuzzy Number (GQFN)}

A new fuzzy number $\tilde{A}=\left(a, b, c, d ; \omega_{1}, \omega_{2}\right)$ is defined as generalized quadrilateral fuzzy number if its membership function is given by

$$
\mu_{\tilde{A}}(x)= \begin{cases}\omega_{1} \frac{(x-a)}{(b-a)}, & a \leq x \leq b \\ \frac{(x-b) \omega_{2}+(c-x) \omega_{1}}{(c-b)}, & b \leq x \leq c \\ \omega_{2} \frac{(x-d)}{(c-d)}, & c \leq x \leq d \\ 0, & \text { otherwise }\end{cases}
$$

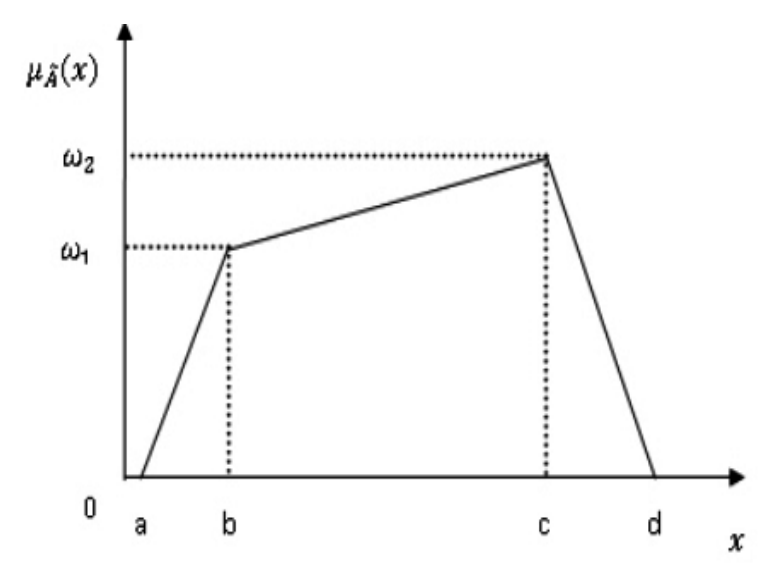

Figure 1. Generalized Quadrilateral Fuzzy Number.

We know that the quadrilateral is the generalization of trapezoid. In our case according to the pictorial representation of the above said fuzzy number, it is not like a trapezoidal as the horizontal lines are not parallel. But, in particular case the two horizontal lines become parallel (i.e. equal weights, $\left.\omega_{1}=\omega_{2}\right)$, then it will become trapezoid. Thus, due to this geographical nature of the particular fuzzy number, it may be called as quadrilateral fuzzy numbers.

\subsection{Arithmetic Operations on GQFN}

In this section, the arithmetic operations between two generalized quadrilateral fuzzy numbers are defined on the universal set of real numbers $\mathfrak{R}$

Let $\tilde{A_{1}}=\left(a_{1}, b_{1}, c_{1}, d_{1} ; \omega_{A_{1}}^{1}, \omega_{A_{1}}^{2}\right)$ and

$\tilde{A_{2}}=\left(a_{2}, b_{2}, c_{2}, d_{2} ; \omega_{A_{2}}^{1}, \omega_{A_{2}}^{2}\right)$

(i) Addition for GQFN:

$\tilde{A_{1}} \oplus \tilde{A_{2}}=\left(a_{1}+a_{2}, b_{1}+b_{2}, c_{1}+c_{2}, d_{1}+d_{2} ; \min \left(\omega_{A_{1}}^{1}, \omega_{A_{2}}^{1}\right)\right.$, $\left.\min \left(\omega_{A_{1}}^{2}, \omega_{A_{2}}^{2}\right)\right)$.

(ii) Subtraction for GQFN:

$$
\begin{aligned}
& \tilde{A_{1}} \ominus \tilde{A_{2}}=\left(a_{1}-d_{2}, b_{1}-c_{2}, c_{1}-b_{2}, d_{1}-a_{2}\right. \\
& \left.\min \left(\omega_{A_{1}}^{1}, \omega_{A_{2}}^{1}\right), \min \left(\omega_{A_{1}}^{2}, \omega_{A_{2}}^{2}\right)\right)
\end{aligned}
$$

(iii) Multiplication for GQFN:

$$
\begin{aligned}
& \tilde{A_{1}} \otimes \tilde{A_{2}}=\left(a_{1} \cdot a_{2}, b_{1} \cdot b_{2}, c_{1} \cdot c_{2}, d_{1} \cdot d_{2} ; \min \left(\omega_{A_{1}}^{1}, \omega_{A_{2}}^{1}\right),\right. \\
& \left.\quad \min \left(\omega_{A_{1}}^{2}, \omega_{A_{2}}^{2}\right)\right)
\end{aligned}
$$

(iv) Scalar Multiplication for GQFN:

$$
\lambda A_{1}= \begin{cases}\lambda a_{1}, \lambda b_{1}, \lambda c_{1}, \lambda d_{1} ;\left(\omega_{A_{1}}^{1}, \omega_{A_{1}}^{1}\right) & \lambda \geq 0 \\ \lambda d_{1}, \lambda c_{1}, \lambda b_{1}, \lambda a_{1} ;\left(\omega_{A_{1}}^{1}, \omega_{A_{1}}^{2}\right) & \lambda<0 .\end{cases}
$$




\subsection{Ranking function for GQFN}

We propose a new ranking function $\mathfrak{R}: F(\mathfrak{R}) \rightarrow \mathfrak{R}$, which maps each fuzzy number into the real number. Let $\tilde{A}=(a, b, c, d$; $\left.\omega_{1}, \omega_{2}\right)$, then

$$
\mathfrak{R}\left(\mathfrak{A}_{\mathbf{1}}\right)=\left(\frac{a_{1}+b_{1}+c_{1}+d_{1}}{4}\right)\left(\frac{\omega_{1}+\omega_{2}}{2}\right)
$$

\section{Fully Fuzzy Transportation Problem}

More number of decision makers are interested to solve fuzzy transportation problems, where all parameters are represented as generalized quadrilateral fuzzy numbers. Let us formulate the fully fuzzy transportation problem as:

$$
\operatorname{Min} z=\sum_{i=1}^{m} \sum_{j=1}^{n} \tilde{c_{i j}} \otimes \tilde{x_{i j}}
$$

subject to

$$
\begin{aligned}
& \sum_{j=1}^{n} \tilde{x_{i j}} \leq \tilde{a_{i}}, i=1,2,3, \ldots, m, \\
& \sum_{i=1}^{m} \tilde{x_{i j}} \leq \tilde{b_{j}}, j=1,2,3, \ldots, n, \\
& \tilde{x_{i j}} \geq 0, \forall i, j
\end{aligned}
$$

\subsection{Algorithm for solving fully fuzzy Transportation problem}

The step by step procedure to solve the fully fuzzy transportation problem, in which all parameters are represented as generalized quadrilateral fuzzy numbers are given as:

Step 1 Check whether the given problem is balanced transportation problem i.e $\sum_{i=1}^{m} a_{i}=\sum_{j=1}^{n} b_{j}$, if not convert it into balanced one by adding dummy row or dummy column.

Step 2 Convert supply and demand into crisp number using ranking function in rows and columns.

Step 3 Find the minimum amount of the fuzzy cost either row or column. Find minimum of $\left(a_{i}, b_{j}\right)$.

case(i): If minimum of $\left(a_{i}, b_{j}\right)=a_{i}$ then allocate $x_{i j}=$ $a_{i}$ in $(i, j)^{t h}$ cell. Ignore $i^{\text {th }}$ row replace $b_{j}$ by $b_{j}-a_{i}$.

case(ii):If minimum of $\left(a_{i}, b_{j}\right)=b_{j}$ then allocate $x_{i j}=$ $b_{j}$ in $(i, j)^{t h}$ cell. Ignore $j^{t h}$ row replace $a_{i}$ by $a_{i}-b_{j}$.

\begin{tabular}{|l|l|l|l|l|l|}
\hline S.NO & $\mathbf{D}_{\mathbf{1}}$ & $\mathbf{D}_{\mathbf{2}}$ & $\mathbf{D}_{\mathbf{3}}$ & $\mathbf{D}_{\mathbf{4}}$ & Supply $\left[a_{i}\right]$ \\
\hline $\mathbf{S}_{\mathbf{1}}$ & $(1,2,3,4 ;$ & $(1,3,4,6 ;$ & $(9,11,12,14 ;$ & $(5,7,8,11 ;$ & $(1,6,7,12 ;$ \\
& $0.6,0.8)$ & $0.6,0.8)$ & $0.6,0.8)$ & $0.6,0.8)$ & $0.6,0.8)$ \\
\hline $\mathbf{S}_{\mathbf{2}}$ & $(0,1,2,4 ;$ & $(-1,0,1,2 ;$ & $(5,6,7,8 ;$ & $(0,1,2,3 ;$ & $(0,1,2,3 ;$ \\
& $0.6,0.8)$ & $0.6,0.8)$ & $0.6,0.8)$ & $0.6,0.8)$ & $0.6,0.8)$ \\
\hline $\mathbf{S}_{\mathbf{3}}$ & $(3,5,6,8 ;$ & $(5,8,9,12 ;$ & $(12,15,16,19 ;$ & $(7,9,10,12 ;$ & $(5,10,12,15 ;$ \\
& $0.6,0.8$ & $0.6,0.8)$ & $0.6,0.8)$ & $0.6,0.8)$ & $0.6,0.8)$ \\
\hline Demand $\left[b_{j}\right]$ & $(5,7,8,10 ;$ & $(-1,5,6,10 ;$ & $(1,3,4,6 ;$ & $(1,2,3,4 ;$ & $(6,17,21,30 ;$ \\
& $0.6,0.8)$ & $0.6,0.8)$ & $0.6,0.8)$ & $0.6,0.8)$ & $0.6,0.8)$ \\
\hline
\end{tabular}

\section{Solution:}

Since $\sum_{i=1}^{m} a_{i}=\sum_{j=1}^{n} b_{j}$ the given problem is balanced fuzzy transportation problem.

\begin{tabular}{|l|l|l|l|l|l|}
\hline S.NO & $\mathbf{D}_{\mathbf{1}}$ & $\mathbf{D}_{\mathbf{2}}$ & $\mathbf{D}_{\mathbf{3}}$ & $\mathbf{D}_{\mathbf{4}}$ & Supply \\
\hline $\mathbf{S}_{\mathbf{1}}$ & {$[(4.55)$} & $(1,3,4,6 ;$ & $(9,11,12,14 ;$ & $(5,7,8,11 ;$ & 4.55 \\
& $(1,2,3,4 ;$ & $0.6,0.8)$ & $0.6,0.8)$ & $0.6,0.8)$ & \\
& $0.6,0.8)]$ & & & & \\
\hline $\mathbf{S}_{\mathbf{2}}$ & $(0,1,2,4 ;$ & $(-1,0,1,2 ;$ & $(5,6,7,8 ;$ & $(0,1,2,3 ;$ & 1.05 \\
& $0.6,0.8)$ & $0.6,0.8)$ & $0.6,0.8)$ & $0.6,0.8)$ & \\
\hline $\mathbf{S}_{\mathbf{3}}$ & $(3,5,6,8 ;$ & $(5,8,9,12 ;$ & $(12,15,16,19 ;$ & $(7,9,10,12 ;$ & 7.35 \\
& $0.6,0.8$ & $0.6,0.8)$ & $0.6,0.8)$ & $0.6,0.8)$ & \\
\hline Demand & 5.25 & 3.5 & 2.45 & 1.75 & 12.95 \\
\hline
\end{tabular}

\begin{tabular}{|l|l|l|l|l|l|}
\hline S.NO & $\mathbf{D}_{\mathbf{1}}$ & $\mathbf{D}_{\mathbf{2}}$ & $\mathbf{D}_{\mathbf{3}}$ & $\mathbf{D}_{\mathbf{4}}$ & Supply \\
\hline $\mathbf{S}_{\mathbf{1}}$ & {$[\mathbf{( 4 . 5 5 )}$} & $(1,3,4,6 ;$ & $(9,11,12,14 ;$ & $(5,7,8,11 ;$ & 0 \\
& $(1,2,3,4 ;$ & $0.6,0.8)$ & $0.6,0.8)$ & $0.6,0.8)$ & \\
& $0.6,0.8)]$ & & & & \\
\hline $\mathbf{S}_{\mathbf{2}}$ & $(0,1,2,4 ;$ & $(-1,0,1,2 ;$ & $(5,6,7,8 ;$ & $(0,1,2,3 ;$ & 1.05 \\
& $0.6,0.8)$ & $0.6,0.8)$ & $0.6,0.8)$ & $0.6,0.8)$ & \\
\hline $\mathbf{S}_{\mathbf{3}}$ & $(3,5,6,8 ;$ & $(5,8,9,12 ;$ & $(12,15,16,19 ;$ & $(7,9,10,12 ;$ & 7.35 \\
& $0.6,0.8$ & $0.6,0.8)$ & $0.6,0.8)$ & $0.6,0.8)$ & \\
\hline Demand & 0.7 & 3.5 & 2.45 & 1.75 & 8.4 \\
\hline
\end{tabular}

\begin{tabular}{|l|l|l|l|l|l|}
\hline S.NO & $\mathbf{D}_{\mathbf{1}}$ & $\mathbf{D}_{\mathbf{2}}$ & $\mathbf{D}_{\mathbf{3}}$ & $\mathbf{D}_{\mathbf{4}}$ & Supply \\
\hline $\mathbf{S}_{\mathbf{1}}$ & {$[\mathbf{( 4 . 5 5 )}$} & $(1,3,4,6 ;$ & $(9,11,12,14 ;$ & $(5,7,8,11 ;$ & 0 \\
& $(1,2,3,4 ;$ & $0.6,0.8)$ & $0.6,0.8)$ & $0.6,0.8)$ & \\
& $0.6,0.8)]$ & & & & \\
\hline $\mathbf{S}_{\mathbf{2}}$ & {$[(\mathbf{0 . 7 )}$} & $(-1,0,1,2 ;$ & $(5,6,7,8 ;$ & $(0,1,2,3 ;$ & 0.35 \\
& $(0,1,2,4 ;$ & $0.6,0.8)$ & $0.6,0.8)$ & $0.6,0.8)$ & \\
& $0.6,0.8)]$ & & & & \\
\hline $\mathbf{S}_{3}$ & $(3,5,6,8 ;$ & $(5,8,9,12 ;$ & $(12,15,16,19 ;$ & $(7,9,10,12 ;$ & 7.35 \\
& $0.6,0.8$ & $0.6,0.8)$ & $0.6,0.8)$ & $0.6,0.8)$ & \\
\hline Demand & 0 & 3.5 & 2.45 & 1.75 & 7.7 \\
\hline
\end{tabular}

\begin{tabular}{|l|l|l|l|l|l|}
\hline S.NO & $\mathbf{D}_{\mathbf{1}}$ & $\mathbf{D}_{\mathbf{2}}$ & $\mathbf{D}_{\mathbf{3}}$ & $\mathbf{D}_{\mathbf{4}}$ & Supply \\
\hline $\mathbf{S}_{\mathbf{1}}$ & {$[\mathbf{( 4 . 5 5 )}$} & $(1,3,4,6 ;$ & $(9,11,12,14 ;$ & $(5,7,8,11 ;$ & 0 \\
& $(1,2,3,4 ;$ & $0.6,0.8)$ & $0.6,0.8)$ & $0.6,0.8)$ & \\
& $0.6,0.8)]$ & & & & \\
\hline $\mathbf{S}_{\mathbf{2}}$ & {$[\mathbf{( 0 . 7 )}$} & {$[\mathbf{( 0 . 3 5 )}$} & $(5,6,7,8 ;$ & $(0,1,2,3 ;$ & 0 \\
& $(0,1,2,4 ;$ & $(-1,0,1,2 ;$ & $0.6,0.8)$ & $0.6,0.8)$ & \\
& $0.6,0.8)]$ & $0.6,0.8)]$ & & & \\
\hline $\mathbf{S}_{\mathbf{3}}$ & $(3,5,6,8 ;$ & $(5,8,9,12 ;$ & $(12,15,16,19 ;$ & $(7,9,10,12 ;$ & 7.35 \\
& $0.6,0.8$ & $0.6,0.8)$ & $0.6,0.8)$ & $0.6,0.8)$ & \\
\hline Demand & 0 & 3.15 & 2.45 & 1.75 & 7.35 \\
\hline
\end{tabular}

\section{Numerical Illustration}

Consider the following fully fuzzy transportation problem. Here the supply, demand and transportation costs are generalized quadrilateral fuzzy numbers.

Step 4 Repeat step 3 until $a_{i}$ and $b_{j}$ are zero. 


\begin{tabular}{|l|l|l|l|l|l|}
\hline S.NO & $\mathbf{D}_{\mathbf{1}}$ & $\mathbf{D}_{\mathbf{2}}$ & $\mathbf{D}_{\mathbf{3}}$ & $\mathbf{D}_{\mathbf{4}}$ & Supply \\
\hline $\mathbf{S}_{\mathbf{1}}$ & {$[\mathbf{( 4 . 5 5 )}$} & $(1,3,4,6 ;$ & $(9,11,12,14 ;$ & $(5,7,8,11 ;$ & 0 \\
& $(1,2,3,4 ;$ & $0.6,0.8)$ & $0.6,0.8)$ & $0.6,0.8)$ & \\
& $0.6,0.8)]$ & & & & \\
\hline $\mathbf{S}_{\mathbf{2}}$ & {$[(\mathbf{0 . 7})$} & {$[(\mathbf{0 . 3 5})$} & $(5,6,7,8 ;$ & $(0,1,2,3 ;$ & 0 \\
& $(0,1,2,4 ;$ & $(-1,0,1,2 ;$ & $0.6,0.8)$ & $0.6,0.8)$ & \\
& $0.6,0.8)]$ & $0.6,0.8)]$ & & & \\
\hline $\mathbf{S}_{\mathbf{3}}$ & & {$[(\mathbf{3 . 1 5})$} & $(12,15,16,19 ;$ & $(7,9,10,12 ;$ & 4.2 \\
& & $(5,8,9,12 ;$ & $0.6,0.8)$ & $0.6,0.8)$ & \\
\hline Demand & 0 & $0.6,0.8)]$ & & & \\
\hline
\end{tabular}

\begin{tabular}{|l|l|l|l|l|l|}
\hline S.NO & $\mathbf{D}_{\mathbf{1}}$ & $\mathbf{D}_{\mathbf{2}}$ & $\mathbf{D}_{\mathbf{3}}$ & $\mathbf{D}_{\mathbf{4}}$ & Supply \\
\hline $\mathbf{S}_{\mathbf{1}}$ & {$[\mathbf{( 4 . 5 5 )}$} & $(1,3,4,6 ;$ & $(9,11,12,14 ;$ & $(5,7,8,11 ;$ & 0 \\
& $(1,2,3,4 ;$ & $0.6,0.8)$ & $0.6,0.8)$ & $0.6,0.8)$ & \\
& $0.6,0.8)]$ & & & & \\
\hline $\mathbf{S}_{\mathbf{2}}$ & {$[\mathbf{( 0 . 7 )}$} & {$[\mathbf{( 0 . 3 5 )}$} & $(5,6,7,8 ;$ & $(0,1,2,3 ;$ & 0 \\
& $(0,1,2,4 ;$ & $(-1,0,1,2 ;$ & $0.6,0.8)$ & $0.6,0.8)$ & \\
& $0.6,0.8)]$ & $0.6,0.8)]$ & & & \\
\hline $\mathbf{S}_{\mathbf{3}}$ & & {$[(\mathbf{3 . 1 5})$} & {$[(\mathbf{2 . 4 5})$} & $(7,9,10,12 ;$ & 1.75 \\
& & $(5,8,9,12 ;$ & $(12,15,16,19 ;$ & $0.6,0.8)$ & \\
& & $0.6,0.8)]$ & $0.6,0.8)]$ & & \\
\hline Demand & 0 & 0 & 0 & 1.75 & 1.75 \\
\hline
\end{tabular}

\begin{tabular}{|l|l|l|l|l|l|}
\hline S.NO & $\mathbf{D}_{\mathbf{1}}$ & $\mathbf{D}_{\mathbf{2}}$ & $\mathbf{D}_{\mathbf{3}}$ & $\mathbf{D}_{\mathbf{4}}$ & Supply \\
\hline $\mathbf{S}_{\mathbf{1}}$ & {$[\mathbf{( 4 . 5 5 )}$} & $(1,3,4,6 ;$ & $(9,11,12,14 ;$ & $(5,7,8,11 ;$ & 0 \\
& $(1,2,3,4 ;$ & $0.6,0.8)$ & $0.6,0.8)$ & $0.6,0.8)$ & \\
& $0.6,0.8)]$ & & & & \\
\hline $\mathbf{S}_{\mathbf{2}}$ & {$[(\mathbf{0 . 7})$} & {$[(\mathbf{0 . 3 5})$} & $(5,6,7,8 ;$ & $(0,1,2,3 ;$ & 0 \\
& $(0,1,2,4 ;$ & $(-1,0,1,2 ;$ & $0.6,0.8)$ & $0.6,0.8)$ & \\
& $0.6,0.8)]$ & $0.6,0.8)]$ & & & \\
\hline $\mathbf{S}_{\mathbf{3}}$ & & {$[(\mathbf{3 . 1 5})$} & {$[(\mathbf{2 . 4 5})$} & {$[(\mathbf{1 . 7 5})$} & 0 \\
& & $(5,8,9,12 ;$ & $(12,15,16,19 ;$ & $(7,9,10,12 ;$ & \\
& & $0.6,0.8)]$ & $0.6,0.8)]$ & $0.6,0.8)]$ & \\
\hline Demand & 0 & 0 & 0 & 0 & 0 \\
\hline
\end{tabular}

Therefore the initial basic feasible solution is

$$
\begin{aligned}
\text { Minimum } z= & (4.55) \times(1,2,3,4 ; 0.6,0.8)+ \\
& (0.7) \times(0,1,2,4 ; 0.6,0.8)+ \\
& (0.35) \times(-1,0,1,2 ; 0.6,0.8)+ \\
& (3.15) \times(5,8,9,12 ; 0.6,0.8)+ \\
& (2.45) \times(12,15,16,19 ; 0.6,0.8)+ \\
& (1.75) \times(7,9,10,12 ; 0.6,0.8) . \\
= & (61.6,87.5,100.45,127.05 ; 0.6,0.8) .
\end{aligned}
$$

The ranking function of $\operatorname{Min} \mathrm{z}=65.905$.

\section{Conclusion}

In this paper, the new notion of fully fuzzy transportation problem with generalized quadrilateral fuzzy numbers is discussed with suitable example. As quadrilateral fuzzy number is a generalization of trapezoidal fuzzy number, the utility of this procedure is more effective than any other procedure of fuzzy transportation problems. This notion can be extended to solve transportation problems with the aid of other fuzzy algorithm.

\section{References}

[1] Amarpreet Kaur and Amit Kumar, A new approach for solving fuzzy transportation problems using generalized trapezoidal fuzzy numbers, Applied Soft Computing, 12( 2012), 1201-1213.

[2] S. Chanas and D. Kutcha, A concept of the optimal solution of the transportation problem with fuzzy cost coefficients, Fuzzy Sets and Systems, 82(1996), 299-305.

[3] M. Venkatachalapathy and A. Edward Samuel, An Alternative Method for Solving Fuzzy Transportation Problem using Ranking Function, International Journal of Applied Mathematical Sciences, 9(1)(2016), 61-68.

[4] A. Nagoor Gani and K. Abdul Rajak, Two stage fuzzy transportation problem, Journal of Physical Sciences, 10(2006), 63-69.

[5] A. Nagoor Gani and S. N. Mohamed Assarudeen, A New Operation on Triangular Fuzzy Number for Solving Fuzzy Linear Programming Problem, Applied Mathematical Sciences, 6(11)(2012), 525-532.

[6] P. Pandian and G. Natarajan, A new algorithm for finding a fuzzy optimal solution for fuzzy transportation problems, Applied Mathematical Sciences, 4(2)(2010), 79-90.

[7] D. Stephen Dinagar and K. Palanivel, The transportation problem in fuzzy environment, International Journal of Algorithms, Computing and Mathematics, 2(2009), 6571.

[8] D. Stephen Dinagar and D. Abirami, On critical path in project scheduling using topsis ranking of more generalized interval valued fuzzy numbers, MJM - An International Journal of Mathematical Sciences with Computer Applications, S(2)(2015) 485-495.

[9] H. J. Zimmermann, Fuzzy programming and linear programming with several objective functions, Fuzzy Sets and Systems, 1(1978), 45-55.

[10] L.A. Zadeh, Fuzzy Sets, Information and Control, 8(1965), 338-353.

[11] L.A. Zadeh, Fuzzy set as a basis for a theory of possibility, Fuzzy Sets and Systems, 1(1978), 3-28.

$$
\text { ISSN(P):2319-3786 }
$$

Malaya Journal of Matematik

ISSN(O):2321 - 5666

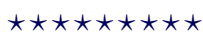

